\title{
Investigation of cadmium telluride films on silicon substrate
}

\author{
V.A. Odarych ${ }^{1}$, A.Z. Sarsembaeva ${ }^{2}$, F.F. Sizov ${ }^{2}$, M.V. Vuichyk ${ }^{2}$ \\ ${ }^{1}$ Taras Shevchenko Kyiv National University, Physics Department, \\ 2, prospect Academician Glushkov,03022 Kyiv, Ukraine, e-mail: wladodarych@narod.ru \\ ${ }^{2} V$. Lashkaryov Institute of Semiconductor Physics, NAS of Ukraine, \\ 45, prospect Nauky, 03028 Kyiv, Ukraine, phone: 5258472; e-mail: sannaz@bigmir.net
}

\begin{abstract}
Properties of cadmium telluride films on silicon substrate, distribution of thickness and refraction index over the sample area were investigated by the ellipsometric method. It was ascertained that the refraction index of cadmium telluride films on a silicon substrate was considerably less than that of monocrystalline CdTe and depends on the film thickness, increasing with the thickness growth.
\end{abstract}

Keywords: cadmium telluride films, ellipsometry, film thickness.

Manuscript received 20.09.05; accepted for publication 25.10.05.

\section{Introduction}

The film technology is the base for development of modern microelectronic devices. In particular, the semiconductor films are used in the solar energy conversion systems, for the signal guidance in optical transmission lines, etc. The thin-film electro-luminescent devices have also been used as emitters in the nearestinfra-red region of spectrum, in particular, those based on cadmium telluride. Actual tasks in this field are the problems of the even distribution of film thickness depending on the sample area, the radiation and the chemical firmness of film structures. To know the properties of the used material, especially the optical ones, is actual for solving the technological problems in many cases.

\section{Investigated samples and experimental details}

We studied CdTe thin films that were obtained using the epitaxy vacuum setup and the "hot wall" method. The monocrystalline silicon was used as a substrate. Before loading into the facility, the silicon substrate was chemically polished in HF acid and then was washed in acetone to remove oxides and contaminations. The CdTe films were formed in the high vacuum $\sim 10^{-7}$ Torr. To obtain various thicknesses, the time of the film deposition was varied, on the samples 1,2 and 3 nominal thicknesses of CdTe epitaxial films were obtained by continuous growth of thin layer during all the time.

The studied samples were thin $(\sim 1 \mathrm{~mm})$ silicon substrates, the central part of which was the film with diameter of spot about $20 \mathrm{~mm}$. The sample 3 had a variable thickness along the radial direction, which was confirmed by interferential colors, from straw-yellow at the edge, through red-brown to dark blue at the central part of the spot. A change of colors in the sample 1 was absent, and the interval of interferential colors of the spot was considerably narrower on the sample 2 . Inhomogeneity in thickness of the investigated samples is an undesirable phenomenon, but for our investigations such inhomogeneity provided favorable conditions to determine parameters of the obtained films.

The ellipsometric investigations were performed at $632.8 \mathrm{~nm}$ wavelength of LEF-3M compensatory zero ellipsometer and at the wavelengths 579, 546, 435, 405 and $366 \mathrm{~nm}$ spectrum of mercury lamp radiation of nonstandard photometric ellipsometer that was calibrated with the help of monocrystalline silicon plate. The operating wavelengths were chosen so that at some of them the silicon and cadmium telluride films would have relatively weak absorption $(632,579$ and $546 \mathrm{~nm})$ and at other wavelengths (435, 405 and $366 \mathrm{~nm}$ ) the mentioned matters would have the strong one.

Measurements were carried out at the fixed angle of incidence $\varphi=60^{\circ}$. Measurements at different wavelengths allow to determine the film thickness and the model of refractive system. Probe light beam was incident on the regions of the investigated film with different interferential colors (up to 7 areas) with different film thicknesses in the probed area, located along the spot radius.

For the used modification of photoelectric ellipsometric method [1], the measured parameters were $\cos \Delta$ and $\operatorname{tg} \psi ;$ where $\Delta$ is the phase difference between $p$ - and $s$-components of an electric vector of reflected light wave; and $\operatorname{tg} \psi$ is the ratio of reflection coefficient in the plane of incidence ( $p$-plane) and in the orthogonal 
$s$-plane. Ellipsometric parameters could be found by measuring the intensities of the radiation reflected from the sample at four azimuths of analyzer of $0^{\circ}, 45^{\circ}, 90^{\circ}$, $-45^{\circ}$ relatively to the plane of incidence and at fixed polarizer azimuths. Before measurements, the ellipsometer was adjusted according to the work [1] and calibrated using a monocrystalline silicon plane to get the optical constants for silicon well-known from reference sources.

\section{Obtained results}

The inverse problem of ellipsometry lays in finding the reflective system parameters, for instance, for one-layer system that would be indexes of absorption and refraction, as well as the layer thickness that would be calculated using measured values of ellipsometric parameters $\psi$ and $\Delta$.

In the case of one-layer system (film + substrate), the basic equation of ellipsometry that relates measured values of the ellipsometric parameter with characteristics of the reflective system is a non-linear transcendent equation, and its analytic solution relatively to optical constants of substrate $n_{3}, \kappa_{3}$ and layer $n_{2}, \kappa_{2}$ doesn't exist. So, the possible way to solve the inverse problem is to increase the number of independent measurements (for instance, in this work, the measurements were performed on the different thickness areas of one sample) followed by calculation of an equation system we have obtained using the least-square method. Complications were related with bad conceding of the ellipsometric function, appearing the false decisions and so on appear on this way.

In our case when the values of optical constants of the substrate (monocrystalline silicon) are well known, only the optical constants $n_{2}, \kappa_{2}$ and thickness $d_{2}$ are to be determined.

In this work, the evident method of measured values diagrams, when the distribution of experimental points obtained at variable values (unknown) of the layer thickness is tried to be described by the adjusted set of investigated system parameters, was used for determination of three unknown film parameters. At the intermediate stages of data calculation, we used the set of ellipsometric programs [2] made by us that contains iteration methods of ellipsometric equation resolving in some particular cases for two-layers reflective system (the intermediate layer thickness is known, upper layer is transparent, etc.).

Results of measurements are presented using the diagrams where measured values of ellipsometric parameters $\cos \Delta$ and $\operatorname{tg} \psi$ are put aside orthogonal axes and a point of the diagram corresponds to each couple of these values.

Results of the measurements carried out at the fixed angle of incidence $\varphi=60^{\circ}$ for different areas are demonstrated in Fig. 1. Values of ellipsometric parameters that are related with the different thickness areas (experimental points) form curves of measured values on diagrams that remind theoretical curves of unchanging values of optical constants; the layer thickness changes along each curve but its optical constants remain fixed. Thus, experimental points for samples 2 and 3 lie on two different curves; this fact testifies that the reflective index is different for layers on these two samples.

The task of calculation of experimental data is to find such values of reflective system parameters that would provide fitting the theoretical curve to the experimental one. In this case, for each experimental point we have to obtain a certain thickness of film.

The refraction index $n_{3}$ and index of absorption $\kappa_{3}$ of monocrystalline silicon of the papers $[3,4]$ were used for calculations. Procedure of choosing the refraction index $n_{2}$ and index of absorption $\kappa_{2}$ of cadmium telluride was performed using the special graphical program that visualizes both experimental and theoretical data as diagrams of measured values that is analogue to those in Fig. 1. In this case, all calculations were made using the software set [2]; the Newton iteration method is the base of this programs.

Results of fitting the theory to the experimental data are presented as curves in Fig. 1. One can see that, for the sample 3, the greater numbers of experimental data are well laid on the theoretical curves; area 1, where the film thickness is the smallest, makes the only exception. For the sample 2, where the film thickness is the most homogenous, all experimental points find their place on the theoretical curve. Experimental point of the sample 1 that has the least thickness among other samples is located near the start point of diagram that presents a substrate without a layer.

Obtained data of optical constants of layers are summarized in Table. Dependences of refraction index of investigated films on the wavelength are depicted in Fig. 2.

From results shown in Fig. 1, we can see that the theoretical curve for the sample 3 well describes the experimental results passing through the experimental points for the greater number of areas; thus the only point for the area 1 over all the wavelengths of the investigated range doesn't find its place on the theoretical curves, it get its place in that region of the diagram $\operatorname{tg} \psi-\cos \Delta$ that is characterized by the smaller refraction indexes than those for the theoretical curve. The film thickness for this area $d_{2}=30 \ldots 40 \mathrm{~nm}$ is estimated by the position of the experimental point. Film optical constants were calculated by the iteration method [2], if the value of the film thickness for this area would be taken as $35 \mathrm{~nm}$. The obtained data for this area are shown in Fig. 2.

Thickness values in the proper areas that were obtained after calculation of ellipsometric data for different wavelengths are identical in the area $\Delta d_{2}= \pm 2 \mathrm{~nm}$, which could be explained as error of the used procedure for the film thickness calculation. 

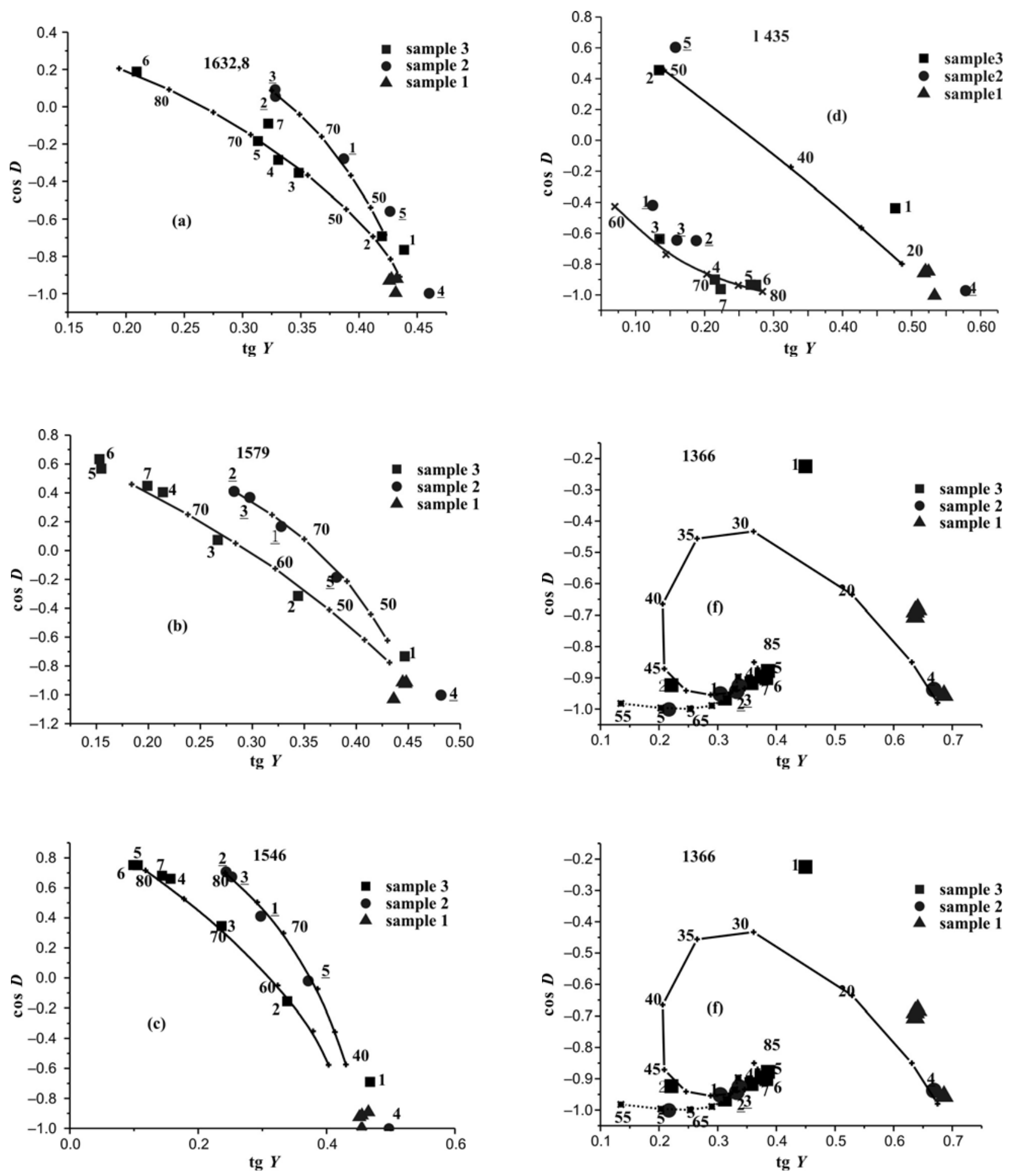

Fig. 1. Values of the ellipsometric parameters $\cos \Delta$ and $\operatorname{tg} \psi$ (points) that were measured at the several wavelengths (a-f) at light reflection from different areas of samples, in comparison with calculated by the equation of ellipsometry in the one-layer model of this reflective system (continuous curves). Area numbers (1-7) are noted near each point, numbers near hyphens on theoretical curves specify the layer thickness in nanometers (parameters of calculation are listed in the table). 
Table. Optical constants $\boldsymbol{n}_{2}$ and $\boldsymbol{k}_{2}$ of investigated samples in comparison with those for CdTe.

\begin{tabular}{|c|c|c|c|c|c|c|c|c|c|c|}
\hline \multirow{2}{*}{$\lambda, \mathrm{nm}$} & \multicolumn{2}{|c|}{ Si [1,2] } & \multicolumn{2}{c|}{ Sample 3 } & \multicolumn{2}{c|}{ Sample 2 } & \multicolumn{2}{c|}{ Sample 1 } & \multicolumn{2}{c|}{ CdTe [3, 4] } \\
\cline { 2 - 11 } & $n_{3}$ & $\kappa_{3}$ & $n_{2}$ & $\kappa_{2}$ & $n_{2}$ & $\kappa_{2}$ & $n_{2}$ & $\kappa_{2}$ & $n_{2}$ & $\kappa_{2}$ \\
\hline 632.8 & 3.876 & 0.028 & 2.14 & 0.43 & 1.99 & 0.42 & $1.7-2.4$ & - & 2.94 & 0.38 \\
\hline 579.065 & 4.04 & 0.03 & 2.20 & 0.36 & 2.015 & 0.39 & $1.7-2.4$ & - & 3.02 & 0.38 \\
\hline 546.07 & 4.08 & 0.042 & 2.15 & 0.38 & 2.02 & 0.36 & $1.7-2.4$ & - & 3.06 & 0.42 \\
\hline 435.834 & 4.56 & 0.185 & 2.44 & 0.37 & 2.20 & 0.35 & $1.5-1.7$ & 0.13 & 3.27 & 0.69 \\
\hline 404.656 & 5.43 & 0.35 & 2.45 & 0.45 & 2.38 & 0.70 & $1.6-1.8$ & 0.12 & 3.39 & 0.90 \\
\hline 366 & 6.52 & 2.705 & 2.61 & 0.895 & 2.373 & 0.62 & 1.86 & 0.29 & 2.9 & 1.53 \\
\hline
\end{tabular}

For the sample 3 that was obtained without a limiting mask, the thickness changes in the range from $35 \mathrm{~nm}$ (the edge of sample) to $81 \mathrm{~nm}$ (center). For the sample 2 where the mask was used, the film thickness increases from $60 \mathrm{~nm}$ at the periphery to $80 \mathrm{~nm}$ at the centre and experimental points for all areas well get their place on the theoretical curve.

The sample 1 has the least thickness, it has no interferential colors. Experimental points that corresponded to various areas are located in that part of the diagram $\operatorname{tg} \psi-\cos \Delta$ that presents a start point of curves for the constant refraction index, by another words, where the substrate is not covered with the layer. Scattering of points for different areas is close to the error of ellipsometric parameter measurements. That's why, the average value was derived at the values $\operatorname{tg} \psi$ and

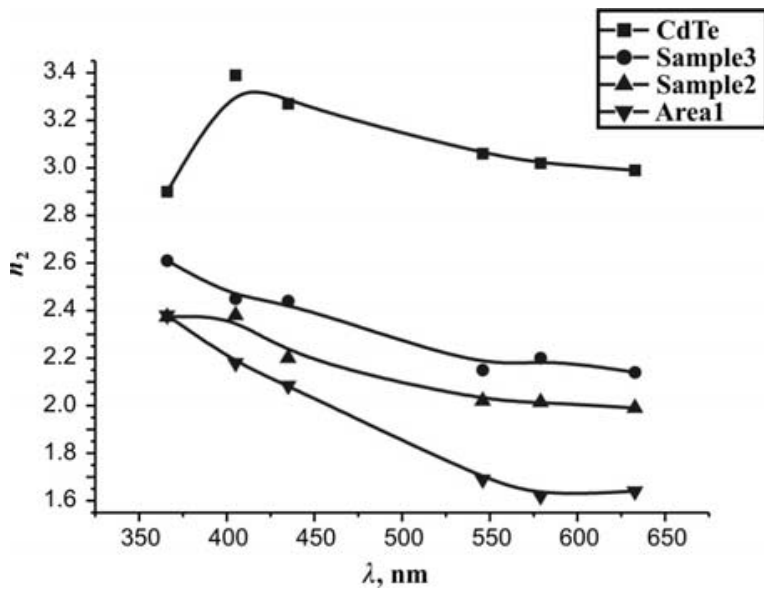

Fig. 2. Spectra of the refraction index of investigated films in comparison with those for monocrystalline cadmium telluride. Film thicknesses $(\mathrm{nm})$ : samples 2 and $3-$ $50 \ldots 80 \mathrm{~nm}$; part 1 (sample 3 ) $-35 \mathrm{~nm}$. $\cos \Delta$ for three areas. By the position on the diagram for $\lambda=632 \ldots 546 \mathrm{~nm}$ (see Fig. 1a, b, c), we estimated the film thickness of this sample as $d_{2}=18 \mathrm{~nm}$ $(15 \ldots 20 \mathrm{~nm})$. Optical constants of the film on this sample were calculated using this thickness value. It should be noted that due to the small thickness of the film, an error of determination of optical constants is large here, so optical parameters were calculated only at some wavelengths where the experimental point was located at a the big distance from the point that presented a substrate.

From these data, one can see that, in the investigated region, the refraction index increases with the wavelength decrease, but its value is considerably smaller than that for monocrystalline cadmium telluride that makes difference from the absorption index which is only a bit less than that for CdTe.

\section{Discussion of results}

The noticeable features of investigated films are: 1) the film optical constants are considerably less than those for monocrystalline cadmium telluride and 2) values of optical constants depend on the film thickness that reduces with its decrease. So for the sample 1, the film thickness is the less $(18 \mathrm{~nm})$ and the refraction index changes on the average within the limits 1.7 to 1.8 in the investigated spectral range. In the area 1 of the sample 3 , where the film thickness reaches $35 \mathrm{~nm}$, the refraction index equals to $1.6-2.38$ and for the sample 3 , where thickness is $60-80 \mathrm{~nm}$, the refraction index increases from 2.14 to 2.61 in the same spectral interval.

In our opinion, these circumstances are caused by the fact that the investigated films are not monocrystalline, but they are porous and have the changed structure that contains pores between the separate grains of the base matter. The pores that are sprinkled in the film bulk 
contain air so the effective refractive index of the film would be less than those for the bulk structure. Obviously the degree of porosity decreases with film thickness increasing, and value of the refraction index that is calculated by the optical methods has to increase.

Such behavior of the refraction index of the film is not strange if to note that the lattice parameters of silicon $(5.4282 \AA)$ and cadmium telluride $(6.477 \AA)$ differ considerably and it's too hard to expect that applied technology for producing the film would form monocrystalline structures based on the given couple of chemical elements.

\section{Conclusion}

It was ascertained that the refraction index of cadmium telluride films is considerably less than that for the monocrystalline CdTe and depends on the film thickness that rise with increasing the latter.
References

1. A.V. Rzanov, K.K. Svitashev, A.I. Semenenko, etc., The base of ellipsometry. Nauka, Novosibirsk (1979) (in Russian).

2. V.A. Odarych, V.I. Panasyuk, V.S. Staschuk // Zhurn. Priklad. Spektr. 56, N 5-6, p. 827-830 (1992) (in Russian); M.A. Byatec, V.T. Kusch, V.A. Odarych, V.I. Panasyuk, Visnyk Kyiv. Univ. Ser. Fiz.Mat. Nauk. N 7, p. 7-12 (1992) (in Ukrainian).

3. V.A. Odarych // Zavodskaya Laboratoriya 43, N 9, p.1093-1095 (1977) (in Russian).

4. V.I. Gavrilenko, A.M. Grekov, D.V. Korbutyak, etc., Semiconductor optical properties. Reference Book. Naukova dumka, Kyiv (1987) (in Russian).

5. D.E. Aspnes, A.A. Studna // Phys. Rev. B 27(2), p. 985-1009 (1983).

6. D.T.F. Marple, H. Ehrenreich // Phys. Rev. Lett. 8(1), p. 87 (1962).

7. Sadao Adachi, Toshifumi Kinura, Norihiro Suzuki // J. Appl. Phys. 74(5), p. 3435-3441 (1993). 